\title{
Benign adult type Osteopetrosis with recurrent osteomyelitis of Maxilla- A rare case report.
}

\author{
Nalini Aswath ${ }^{1}$ and Bhargavi Ramesh ${ }^{1}$ \\ ${ }^{1}$ Sree Balaji Dental College and Hospital
}

February 19, 2022

\begin{abstract}
Osteopetrosis is a genetic bone disease. The osteoclastic cells responsible for bone resorption are defective and non-functional. The bones are dense with loss of cortico medullary differentiation. Patient is prone for repetitive fractures of bone. A case report of osteopetrosis with osteomyelitis of maxilla consequent to dental extraction is presented.
\end{abstract}

\section{Introduction:}

Osteopetrosis, also known as the marble bone disease was first described by Albers-Schönberg, a German radiologist in $1904 .{ }^{1}$ It is a rare metabolic bone disorder characterised by generalised sclerosis of the skeletal system followed by compressive neuropathies usually leading to hearing loss and visual impairment. Hepatosplenomegaly and anaemia are important associated clinical features. The genetic component of the disease consists of numerous mutations that can result in different clinical forms of the disease. It can manifest as an accidental radiographic finding or as a life- threatening type associated with bone marrow failure in infants. ${ }^{2}$ The adult type of the disease is usually diagnosed through accidental radiographic findings and doesn't require intervention. Any bone invasive intervention for other medical conditions can be debilitating in these patients. This case report is of one such rare autosomal dominant benign adult type osteopetrosis patient who developed osteomyelitis of maxilla following a dental extraction.

\section{Case report:}

A 47-year-old male patient reported to our outpatient department with the chief complaint of intermittent pain and swelling in the right upper back tooth region for the past 1 week. He was a known case of type 2 diabetes for the past two years and was under treatment. Patient had history of multiple fractures of long bones. He had also undergone dental extraction of right maxillary posterior teeth, a year ago that was eventful and proceeded to osteomyelitis of maxilla. Patient was then admitted and underwent sequestrectomy under general anasthesia. A year later he had come with complaints of recurrence of pain in the surgical area.

On general examination the patient had short stature with frontal bossing and flattening of the nasal bridge, hypertelorism, exophthalmos with proptosis and strabismus of the left eye. (Fig 1) The patient gave history of apparent hearing loss on the left side with complete loss of sight in the left eye. Extra oral examination revealed mild swelling in the right lateral aspect of the face approximately $1 \mathrm{~cm}$ below the lower eyelid and $2 \mathrm{cms}$ above the upper lip that was soft and tender on palpation.

Intraoral examination showed edentulous area in relation to right maxillary posterior region with missing premolars and molars. Early periodontal changes were observed with generalised loss of gingival attachment. There was evidence of sinus opening with yellowish white pus discharge in the edentulous 14 region. Surgical 
scarring was noted with loss of alveolar ridge along the right maxillary posterior edentulous area. (Fig 2) Tenderness was present on palpation of the affected region.

Orthopantomogram revealed presence of unhealed socket in right maxillary posterior region with patchy areas of radiolucency. There was evidence of generalised dense amorphous radio-opacities involving the maxilla and mandible along the periapical region of the teeth with altered trabecular pattern. (Fig 3)

The skull x- ray images revealed marked density of the calvaria, base of the skull and the facial bones suggestive of generalised sclerosis of skull bones. The lateral skull x-ray showed increased sclerosis of bones with altered "bone in bone" appearance of the vertebra. (Fig 4) The patient was then further advised for general body X-rays in which the spine view exhibited typical "sandwich" vertebrae and AP view of pelvis bone showed loss of cortico-medullary differentiation with altered femoral neck angle. (Fig 5)

The computed tomography evaluation included maxillary hypoplasia with aplastic/ hypoplastic frontal and sphenoidal sinuses. Nerve compression along a torturous left optic nerve was identified with bilateral optic canal narrowing present. (Fig 6) The cranial bones appeared to be thick, hyper sclerotic with loss of corticomedullary differentiation. At the infected right maxillary posterior region, there was soft tissue density noted with few calcific specks within the right maxillary sinus associated with bony thickening.

USG abdomen was done to rule out hepatosplenomegaly. Blood evaluation reported mild anaemia with normal levels of serum calcium $(9.1 \mathrm{mg} / \mathrm{dl})$, phosphorous $(2.6 \mathrm{mg} / \mathrm{dl})$ and Alkaline phosphatase (96 IU/L). However, the total serum acid phosphatase levels were elevated $(2.60 \mathrm{U} / \mathrm{L})$. Corelating all the findings, a clinico-radiological diagnosis of Osteopetrosis with recurrent chronic osteomyelitis of right maxilla was given. Patient was treated with oral antibiotics and is on long term follow up.

\section{Discussion:}

Osteopetrosis is a rare inheritable bone disorder characterised by sclerosis of the skeletal system. The osteoclastic activity in the bone is defective or dysfunctional due to an inherent genetic defect or a change in the microenvironment of the osteoclast that interferes with its function. This is a rare medical condition that occurs 1 in 2,50,000 live births. ${ }^{2}$ Clinically the disease has been characterised into three types as given below. Infantile or malignant osteopetrosis - Autosomal recessive type, An intermediate autosomal recessive type and

Autosomal dominant "benign" type - Adults. The autosomal recessive type of osteopetrosis that occurs in infancy is usually lethal. The affected child demonstrates a failure to thrive due to numerous complications associated with bone remodelling. The intermediate recessive type occurs without major lethal complications, yet the quality of life appears to be questionable. Associated features of compressive neuropathies, bone deformities, bone pain and anemia remains evident. The benign adult type of osteopetrosis is usually diagnosed as an accidental finding on the radiographs following multiple bone fractures. These patients tend to have a relatively normal life and develop complications as they age. The continuous bone deposition without resorption leads to occlusion of nerve canals causing nerve atrophy. ${ }^{3}$ Among osteopetrosis patients, occurrence of benign adult type autosomal disease is more common, though the frequency of the disease occurrence as such is rare. When present, the symptoms are usually seen as bone pain or increasing tendency to fracture. It is important from a clinical perspective to investigate for osteopetrosis in patients who report with increased frequency of fractures while considering other possible conditions such as osteopenia and osteoporosis.

Genetic aspect of the Adult type osteopetrosis disease is been associated with loss of functional mutations in almost 8 identified genes isolated to $1 \mathrm{p} 21$ chromosome. The osteoclastic cells that are responsible for bone resorption are defective and non-functional. The infantile autosomal recessive type is identified to be associated with mutation of TNFSF11 and TNFRSF11a genes that cause a defect along the ruffled borders of osteoclastic cell. The intermediate autosomal recessive type has mutation of CAII gene that produces carbonic anhydrase enzyme. This enzyme plays a pivotal role in bone resorption and failure to produce the enzyme leads to non-functional osteoclasts. The adult autosomal dominant type is caused by mutation to 
CLCN7 gene that disrupts the chloride channel functioning in the osteoclastic cells. ${ }^{4}$ Failure of effective bone resorption leads to encroachment of the cortical bone into the medullary spaces. The insufficient medullary cavity is inappropriate for haematopoiesis. Therefore, a simultaneous occurrence of haematological abnormalities including leukopenia and anaemia is inevitable. This haematological insufficiency is compensated by extramedullary haematopoiesis which eventually results in hepatosplenomegaly in the affected individuals. ${ }^{5}$

The clinical complications of the disease include back pain with or without scoliosis, lower limb deformities resembling rickets, increased tendency to fracture including pathological bone fractures, nerve palsy, otitis media, oral infections ranging from simple dental caries with odontogenic abscess to invasive osteomyelitis of the jaw, osteoarthritis of major joints, optic nerve atrophy. Most commonly fractures are seen in femur followed by ribs, tibia, clavicle, cranial bones and then mandible. ${ }^{6}$

Our patient here, had previous fracture of femur that were evident on the radiographs. The other characteristic radiographic findings of the disease are diffuse sclerosis of bone with loss of corticomedullary differentiation, loss of femoral neck angle, endplate sclerosis of vertebra seen as "rugger jersey" spine, metaphyseal deformity of long bones which was seen as flask shape called "Erlenmeyer-flask" deformity and occlusion of nerve canals. ${ }^{7}$ All these features were evident in this patient.

The head and neck manifestation of the disease includes dolichocephalic head with frontal bossing, proptosis and hypertelorism. Facial nerve palsy can also be seen. ${ }^{8}$ Our patient showed all these signs except for facial nerve palsy. The signs of nerve compression in this patient was identified by optometric test revealing complete loss of vision on left eye and history of loss of hearing on the left ear.

Dental manifestation of osteopetrosis include disturbance of tooth eruption due to impaired alveolar bone resorption by osteoclasts leading to partial anodontia, hypodontia, malformed teeth with smaller pulp chambers, multiple carious teeth, enamel hypoplasia, Hypercementosis, early onset periodontitis. The increased bone density, initially presented as thickening of lamina dura, obscures the roots. ${ }^{9}$ Our patient here, had a carious maxillary right molar tooth which was extracted that lead to unhealed socket causing osteomyelitis. It was further treated by sequestrectomy that went unresolved over a year. For such patients, a more conservative management like endodontic therapy for a carious tooth should be advised. Extraction and other surgical procedures almost always develop complications due to dysregulated bone metabolism. ${ }^{10}$

The usual treatment options include hematopoietic stem cell transplantation (HSCT) which may not be a success in many cases. As osteoclastic cells arise from hematopoietic cell line, HSCT may show success, yet, inherent genetic mutation cannot be changed. When complications arise, surgical management can be considered given the risk- benefit ratio tailored to the individual patient. Surgical complications include sepsis and non-union of the bones following a fracture. Other practical difficulties such as fragile yet hard bone substance poses a greater challenge to the surgeons. ${ }^{6}$ Other supportive and conservative management include pharmacotherapy with corticosteroids, bisphosphonates, Vitamin D3 supplements. Management of osteomyelitis in patients with osteopetrosis is complicated. Primary therapeutic approach is generally a course of antibiotics with complete debridement and closure of the infected soft tissue. Hyperbaric oxygen therapy has been recommended for management of cases chronic osteomyelitis. ${ }^{7}$ Our patient was treated with a course of antibiotics and is under regular follow-up.

\section{Conclusion:}

Osteopetrosis is a rare, unpreventable and untreatable disease. The disease is complicated enough that even a regular procedure of dental extraction can lead to a debilitating onset of osteomyelitis. Though the occurrence of osteomyelitis is reported to be higher in mandible compared to maxilla, this patient reported with a rare case of osteomyelitis of maxilla. This can only be explained by the severity of the nature of the disease. When a simple orthopantomogram can reveal such a dreadful disease, care must be taken to anticipate such conditions, at least in patients suspected of bone disorders. The bone disorders usually disrupt the entire skeletal system which will almost always clinically manifest in the patients' physical appearance. Better application of this knowledge along with cautious evaluation can definitely be beneficial to every such patient who report to us. 


\section{References:}

1. Albers-Schönberg HE. 1904. Röntgenbilder einer seltenen Knockenerkrankung. Munch Med Wochenschr 5:365-8.

2. Kant P, Sharda N, Bhowate RR. Clinical and radiological findings of autosomal dominant osteopetrosis type II: a case report. Case reports in dentistry. https://doi.org/10.1155/2013/707343

3. Van Hul W, Bollerslev J, Gram J, Van Hul E, Wuyts W, Benichou O, Vanhoenacker F, Willems PJ.1997. Localization of a gene for autosomal dominant osteopetrosis (Albers-Schönberg disease) to chromosome 1p21. Am J Hum Genet 61(2): 363-369.

4. Bailey JR, Tapscott DC. 2021 Osteopetrosis. Treasure Island (FL): StatPearls Publishing Bookshelf ID: NBK557529 PMID: 32491461

5. Yamada T, Mishima K, Imura H, Ueno T, Matsumura T, Moritani N, Sugahara T. 2009.Osteomyelitis of the mandible secondary to infantile osteopetrosis: A case report. Oral Surgery, Oral Medicine, Oral Pathology, Oral Radiology, and Endodontology.107(6):e25-9.

6. Benichou OD, Laredo JD, De Vernejoul MC. 2000.Type II autosomal dominant osteopetrosis (AlbersSchönberg disease): clinical and radiological manifestations in 42 patients. Bone 26(1):87-93.

7. Verma P, Kadam S, Umarji HR, Surya V. 2014. A rare case report of intermediate osteopetrosis and review of literature. Journal of Cleft Lip Palate and Craniofacial Anomalies 1(2):127.

8. Wong ML, Balkany TJ, Reeves J, Jafek BW.1978. Head and neck manifestations of malignant osteopetrosis. Otolaryngology 86(4suppl):ORL-585.

9. Jayachandran S, Preethi M.2018. Clinical spectrum of osteopetrosis with secondary osteomyelitis of the mandible: Report of two cases. Journal of Indian Academy of Oral Medicine and Radiology. 30(2):165.

10. Mohn A, Capanna R, Morgese G, Chiarelli F. 2004. Autosomal malignant osteopetrosis. From diagnosis to therapy. Minerva pediatrica 56(1):115-8. 


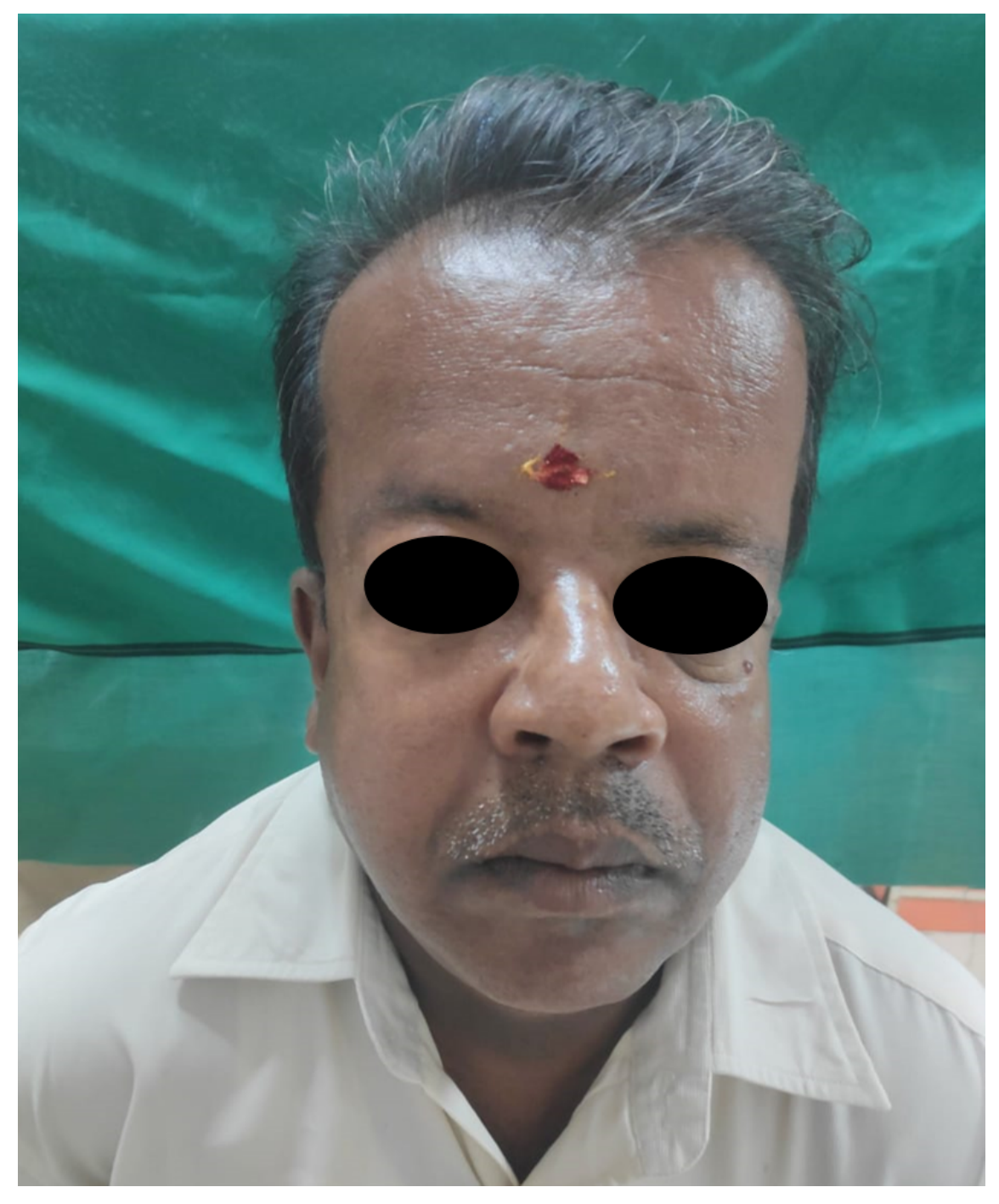



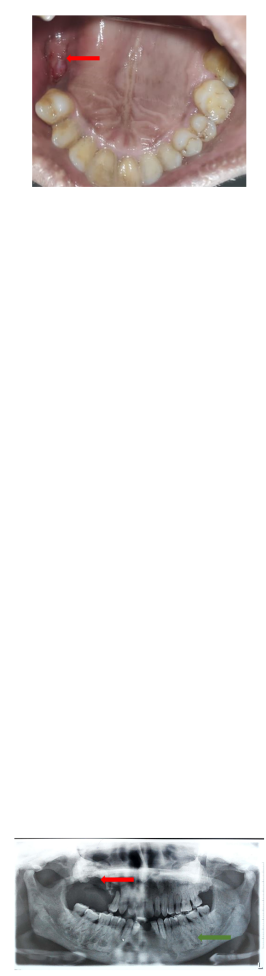

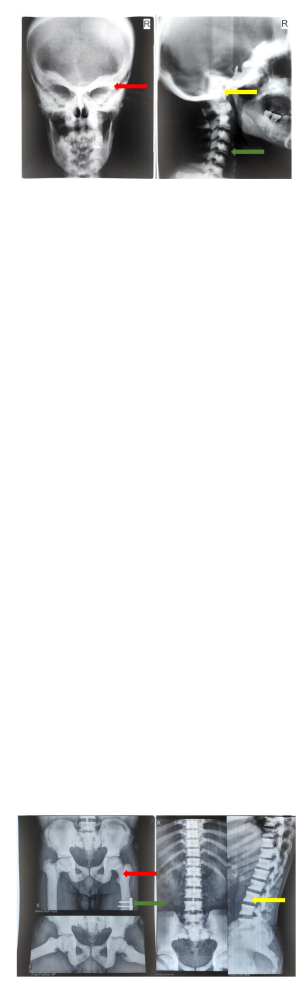


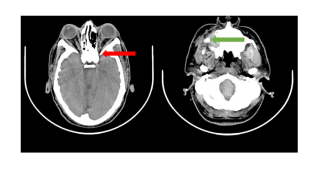

